\title{
Erratum to: How to build an infinite lottery machine
}

\author{
John D. Norton ${ }^{1}$
}

Published online: 20 June 2017

(C) Springer Science+Business Media Dordrecht 2017

\section{Erratum to: Euro Jnl Phil Sci \\ DOI 10.1007/s131940170174-4}

The original version of this article unfortunately contained a mistake. Formulae in footnotes 11, 12 and in Section 11.3 Nonmeasurable outcomes, 3rd sentence, paragraph 5, were incorrectly presented. The corrected formulae are given below.

Footnote 11, 2nd sentence

The result of two half steps is $+1,-1$ or 0 , conforming with the probabilities of (4). In $N$ steps $=2 N$ half-steps, the probability of $m$ half steps up and $2 N-m$ half steps down is just $\frac{(2 N) !}{m !(2 N-m) !} \frac{1}{2^{2 N}}$. This results in a net motion of $m-(2 N-m)=2(m-N)$ half steps up, which corresponds to the walker moving to cell $n=(m-N)$. This last probability becomes equation (5) with the substitutions $m=N+n$ and $2 N-m=2 N-(n+N)=N-n$.

Footnote 12

That is, for any $m, n, \lim _{N \rightarrow \infty} \frac{P(m, N)}{P(n, N)}=\frac{(N+n) !(N-n) !}{(N+m) !(N-m) !}=1$.

3rd sentence, paragraph 5 in Section 11.3 Nonmeasurable outcomes

The probability that the first $N$ terms of a row that encodes some nominated finite number is not a truncated row in the rectangle is $\left(1-1 / 2^{N}\right)^{M}=\left(1-1 / 2^{N}\right)^{2^{N} K}$.

The original article was corrected.

The online version of the original article can be found at http://dx.doi.org/10.1007/s13194-017-0174-4

John D. Norton

jdnorton@pitt.edu; http://www.pitt.edu/ jdnorton

1 Department of History and Philosophy of Science, University of Pittsburgh, Pittsburgh, PA, USA 